ARTICLE

Received 6 Aug 2014 | Accepted 5 Sep 2014 | Published 10 Oct $2014 \quad$ DOl: 10.1038/ncomms6166

\title{
Inflammation-driven carcinogenesis is mediated through STING
}

Jeonghyun Ahn ${ }^{1, \star}$, Tianli Xia ${ }^{1, \star}$, Hiroyasu Konno ${ }^{1}$, Keiko Konno ${ }^{1}$, Phillip Ruiz ${ }^{2} \&$ Glen N. Barber ${ }^{1}$

Chronic stimulation of innate immune pathways by microbial agents or damaged tissue is known to promote inflammation-driven tumorigenesis by mechanisms that are not well understood. Here we demonstrate that mutagenic 7,12-dimethylbenz(a)anthracene (DMBA), cisplatin and etoposide induce nuclear DNA leakage into the cytosol that intrinsically activates stimulator of interferon genes (STING)-dependent cytokine production. Inflammatory cytokine levels are subsequently augmented in a STING-dependent extrinsic manner by infiltrating phagocytes purging dying cells. Consequently, STING ${ }^{-/-}$mice, or wild-type mice adoptively transferred with STING ${ }^{-/-}$bone marrow, are almost completely resistant to DMBA-induced skin carcinogenesis compared with their wild-type counterparts. Our data establish a role for STING in the control of cancer, shed significant insight into the causes of inflammation-driven carcinogenesis and may provide a basis for therapeutic strategies to help prevent malignant disease.

\footnotetext{
${ }^{1}$ Department of Cell Biology and Sylvester Comprehensive Cancer Center, University of Miami School of Medicine, Miami, Florida 33136, USA. ${ }^{2}$ Department of Surgery, University of Miami School of Medicine, Miami, Florida 33136, USA. * These authors contributed equally to this work. Correspondence and requests for materials should be addressed to G.N.B. (email: gbarber@med.miami.edu).
} 
T he innate immune response is essential for protection of the host against microbial infection, although chronic stimulation of these pathways can cause unwarranted inflammatory disease including systemic lupus erythematosus (SLE) and even cancer ${ }^{1-3}$. While the toll-like receptor and RIG-Ilike (RLR) pathways have been clearly shown to be important in facilitating innate immune signalling processes in response to pathogens, they have not been found to be notably responsible for manifesting inflammatory disorders induced by self-DNA ${ }^{4}$. Recently, however, a cellular sensor referred to as STING (for stimulator of interferon (IFN) genes) has been shown to control innate immune gene activation in response to cytosolic DNA generated by DNA pathogens, necrotic or inappropriately apoptosed cells $s^{5-7}$. Whereas STING is essential for production of type I IFN following pathogen infection of the cell, chronic STING activity has been found to lead to lethal inflammatory disease in model systems and may be responsible for AicardiGoutieres syndrome (AGS) and plausibly other disorders in human patients ${ }^{5,8}$.

\section{Results}

DMBA induces cytokine production in a STING-dependent manner. Inflammation triggered by infectious disease or environmental carcinogens is also considered to play a key role in tumour development, although the mechanisms remain to be fully elucidated ${ }^{1,2}$. Given this and the importance of STING in mediating cytokine production, we therefore queried the plausible role of STING in inflammation-driven carcinogenesis. To facilitate this, we utilized the carcinogen 7,12-dimethylbenz(a) anthracene (DMBA), a polyaromatic hydrocarbon (PAH) known to induce cutaneous tumours when applied to the skin of mice 9 . $\mathrm{PAHs}$ are currently classified as human mutagens and have been shown to have a carcinogenesis effect in humans ${ }^{10}$. DMBA is known to activate cytokine-driven MyD88 signalling and tumour necrosis factor alpha $(\mathrm{TNF} \alpha)$ production, which facilitates tumorigenesis, although the initiating responsible pathway remains unknown ${ }^{11,12}$. We thus treated wild-type (WT) mouse embryonic fibroblasts (MEFs) or STING-deficient MEFs (SKO) with DMBA and examined cytokine production by these cells. Principally, we observed that DMBA clearly triggered STING translocation from the endoplasmic reticulum (ER) to perinuclear, non-ER microsome compartments in the cells (Fig. 1a). The translocation of STING via autophagy is essential to invoke STING-dependent signalling ${ }^{13,14}$. We further demonstrated that DMBA induced STING phosphorylation, indicated by an increase in the molecular weight of STING ${ }^{14}$, an event also required for STING activity (Fig. 1a). Further, microarray analysis of DMBA-treated WT cells indicated a wide variety of cytokine and chemokine production, including IL1, ICAM1, IL11, PTGES and TNF $\alpha$, which are known proinflammatory agents (Fig. 1b; Supplementary Table 1). Significantly, the induction of these genes by DMBA was markedly reduced in SKO MEFs, indicating that their production involved the STING pathway (Fig. 1b; Supplementary Table 1). To investigate further, we similarly treated MEFs lacking functional DNaseIII (Trex1) expression with DMBA. Trex1 may function, at least in part, to degrade STING agonists such as leaked DNA in the cytoplasm, since loss of Trexl function in mice causes STING-dependent lethal inflammatory diseases $8,15,16$. Mutations in Trex1 have also been found in patients with AGS and severe $S_{L E}{ }^{17}$. We postulated that the effects of DMBA may be profoundly accentuated in the absence of Trex1. This analysis indeed confirmed that DMBAtreated MEFs lacking Trexl (TKO) had even more augmented cytokine production compared with WT MEFs (Fig. 1b;
Supplementary Table 1). This was verified using quantitative real-time PCR (qPCR) analysis and enzyme-linked immunosorbent assay (ELISA) to demonstrate a comprehensive increase in type I IFN production in DMBA-treated cells lacking Trex1, events that were almost completely suppressed in the absence of STING (Fig. 1c; Supplementary Fig. 1a,b). Accordingly, we observed STING-dependent activation and nuclear translocation of both NF- $\kappa$ B and IRF3 in DMBA-treated WT and TKO MEFs, but not in cells lacking STING (Fig. 1d; Supplementary Fig. 1c-f). Given this, we next considered whether other DNA adductforming agents, including cisplatin and etoposide, were similarly capable of stimulating STING-dependent cytokine activation comparable to DMBA (Supplementary Table 2$)^{18}$. This analysis, carried out on MEFs and primary human keratinocytes, confirmed that a variety of DNA-damaging agents are able to stimulate cytokine production in a STING-dependent manner (Fig. 1e,f; Supplementary Fig. 2a-f). Similar to loss of STING, we observed that knockdown of cGAS, a cyclic guanosine monophosphate-adenosine monophosphate synthase that produces non-canonical cyclic dinucleotides capable of activating STING ${ }^{19}$, also greatly reduced the production of inflammatory cytokines triggered by DMBA as well as by other DNA-damaging agents, such as cisplatin and camptothecin (Fig. 1g; Supplementary Fig. 2g,h). Thus, the mutagens, DMBA, cisplatin and etoposide, generate intrinsic inflammatory effects through the activation of STING-dependent signalling.

DMBA causes cytoplasmic DNA leakage independent of STING. STING is known to control the induction of cytokines by aberrant cytosolic DNA species ${ }^{6,7}$. We noted that it has previously been shown that cell death invoked by etoposide can cause nucleosome leakage into the cytoplasm, plausibly generated by single- or double-strand breaks of DNA promoted by DNA adduct formation ${ }^{20}$. The presence of nuclear DNA in the cytoplasm could conceivably activate the STING pathway and generate cytokine production. Initially, we observed that DMBAtreated MEF cells underwent slightly more cell death over a period of 3 days, as determined by trypan blue analysis and Annexin V staining (Supplementary Fig. 3a,b). We concomitantly examined DMBA-treated MEFs and examined the cells for the presence of cytoplasmic DNA. This approach showed significant DNA leakage in the cytoplasm of DMBA-, cisplatinor etoposide-treated MEFs, but not mock-treated MEFs, as determined by fluorescence in situ hybridization analysis, antidouble-stranded DNA (dsDNA) antibody as well as PicoGreen staining (a dsDNA stain), (Fig. 2a-d; Supplementary Fig. 3c,d). A similar effect was seen in MEF cells lacking STING, indicating that this event was STING independent (Fig. 2a-d; Supplementary Fig. 3c,d). To extend this analysis further, we examined, by two approaches, whether DMBA-treated cells also exhibited an increase in the presence of extranuclear histones. As determined by fractionation analysis of cytoplasmic cell extracts treated with or without DMBA or ultraviolet irradiation, DMBAtreated MEFs indeed displayed the presence of cytoplasmic histones (histone H3) (Fig. 2e). Fluorescence microscopy analysis of histone $\mathrm{H} 3$ staining confirmed this study (Supplementary Fig. 3e,f). Moreover, Trex $1^{-/}$MEFs seemed to exhibit more histone $\mathrm{H} 3$ in the cytoplasm, as determined by this assay, compared with WT MEFs, even under untreated conditions (Fig. 2e; Supplementary Fig. 3e,f). A similar effect was observed in MEFs lacking STING, again indicating that extranuclear nucleosome production does not require STING (Fig. 2e; Supplementary Fig. 3e,f). Electron microscopy using goldlabelled antibody confirmed the presence of histones and dsDNA species in the cytoplasm of DMBA-treated MEF cells 
a
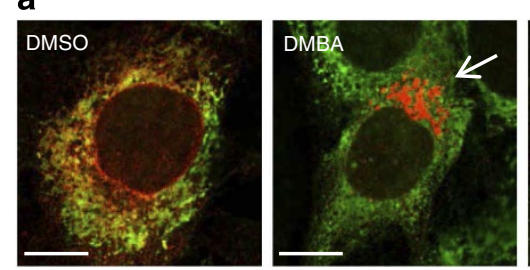

b

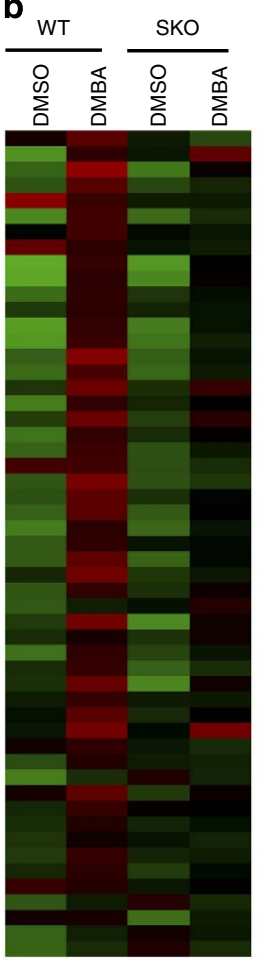

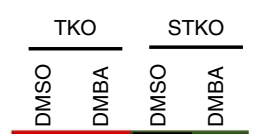
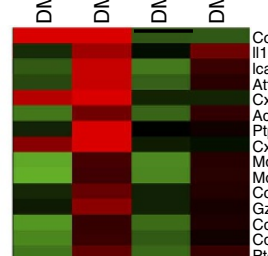

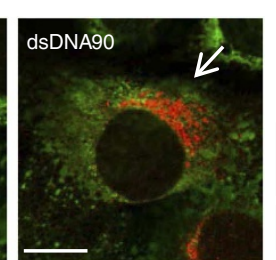

C
STING-HA Calreticulin
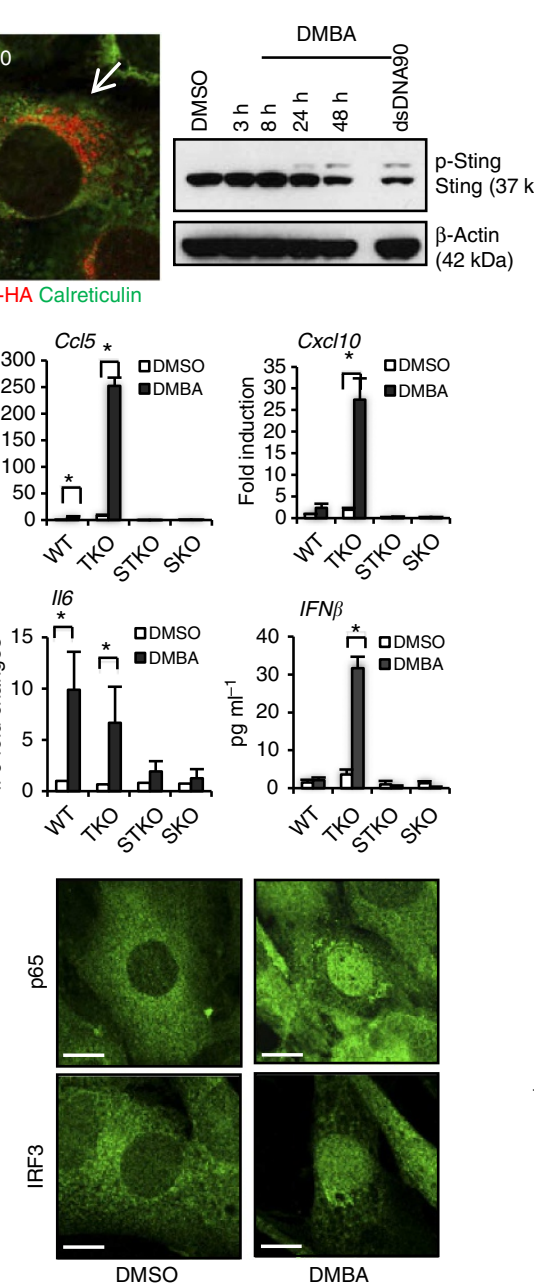

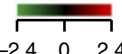

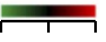

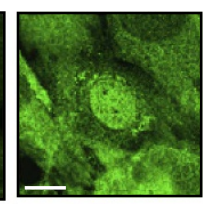

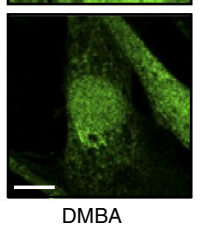

e

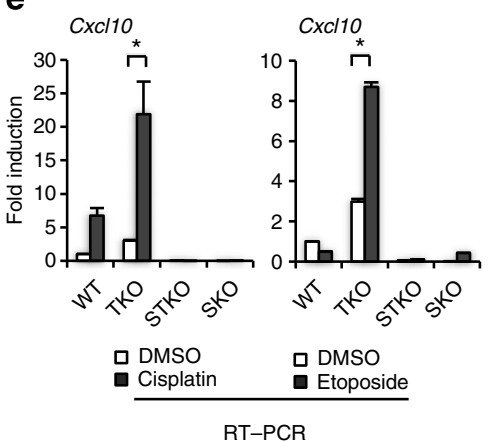

f

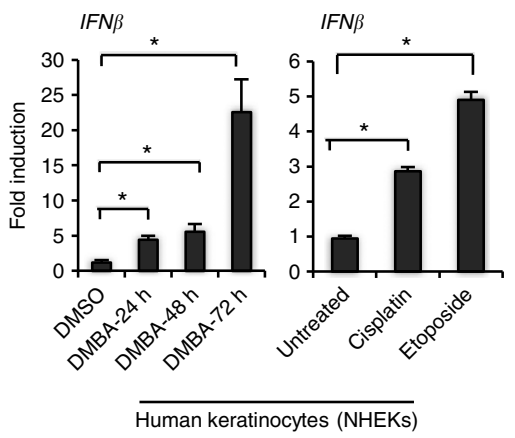

g

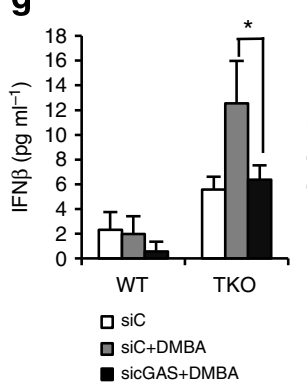

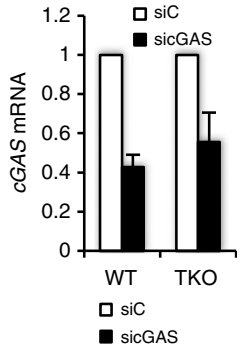

Figure 1 | The role of STING in DMBA-induced cytokine production. (a) WT MEFs expressing STING-HA were either transfected with $3 \mu \mathrm{g} \mathrm{I}^{-1}$ of dsDNA90 for $3 \mathrm{~h}$ or treated with $20 \mu \mathrm{g} \mathrm{ml}{ }^{-1}$ of DMBA. DMSO treatment was included as control. Using HA antibody for STING and anti-calreticulin for ER marker, immunofluorescence microscopy analysis was performed $48 \mathrm{~h}$ after DMBA treatment (left, original magnification at $\times 1,260$; bar size, $10 \mu \mathrm{m}$ ) and immunoblot analysis was performed at the designated time points (right), (also see Supplementary Fig. 10). p-Sting, phosphorylated STING. (b) Gene array analysis of WT, TKO, STKO and SKO MEFs treated with DMBA at $20 \mu \mathrm{g} \mathrm{ml} \mathrm{m}^{-1}$ or DMSO as control for $48 \mathrm{~h}$. Highest variable inflammation-related genes are shown. Rows represent individual genes; columns represent individual samples. Pseudo-colours indicate transcript levels below (green), equal to (black) or above (red) the mean. Scale represents the intensity of gene expression (log2 scale ranges between -2.4 and 2.4). (c) Quantitative PCR (qPCR) analysis and IFN $\beta$ ELISA assay of MEFs treated with DMBA same as in b. (d) Immunofluorescence microscopy analysis of p65 and IRF3 in WT MEFs treated with DMBA same as in $\mathbf{b}$. Original magnification, $\times 1,260$; bar size, $10 \mu \mathrm{m}$. (e) qPCR analysis of WT, TKO, STKO and SKO MEFs treated with $10 \mu \mathrm{M}$ of cisplatin or $250 \mu \mathrm{M}$ of etoposide for $48 \mathrm{~h}$. DMSO treatment was included as controls. (f) qPCR analysis of IFN $\beta$ messenger RNA in human keratinocytes treated with $20 \mu \mathrm{g} \mathrm{ml} \mathrm{l}^{-1}$ of DMBA for 24,48 or $72 \mathrm{~h}$ or with $10 \mu \mathrm{M}$ of cisplatin or $250 \mu \mathrm{M}$ of etoposide for $48 \mathrm{~h}$. (g) WT and TKO MEFs were transfected with cGAS or control small interfering RNA (siRNA) for $48 \mathrm{~h}$ followed by DMBA treatment same as in $\mathbf{b}$, and were then subjected to the IFN $\beta$ ELISA assay. cGAS expression level after siRNA treatment was determined by qPCR. Data are representative of at least two independent experiments. Error bars indicate s.d. ${ }^{\star} P<0.05$, Student's $t$-test. WT; Sting ${ }^{+/+}$Trex $1^{+/+}$, SKO; Sting ${ }^{-/-}$Trex $1^{+/+}, \mathrm{TKO}$; Sting ${ }^{+/+}$, Trex $1^{-/-}$, STKO; Sting ${ }^{-/-}, \operatorname{Trex1^{-/-}}$.

(Fig. 2f; Supplementary Fig. 3g,h). The presence of histones/ dsDNA was found to be increased in TKO MEFs treated with DMBA. However, even untreated TKO cells appeared to have slightly increased histone/dsDNA complexes in the cytoplasm compared with WT MEFs (Fig. 2; Supplementary Fig. 3c-h). Plausibly, Trex1 may function to help eliminate the presence of such leaked DNA into the cytoplasm. Collectively, our data indicate that DMBA may facilitate DNA leakage, a consequence that can lead to the activation of the STING pathway, an effect augmented by loss of Trex1.
STING $^{-/-}$mice are resistant to DMBA skin tumorigenesis. DMBA is used as a mutagen in tumorigenic studies and is known to induce inflammation-driven epithelial cancer when applied to the skin of mice, although the initiating pathway remains to be determined $^{2,9}$. Given that we had observed that DMBA induces cytokine production in normal fibroblasts in a STING-dependent manner, we treated WT or STING-deficient mice (SKO) on a C57BL/6 background with $10 \mu \mathrm{g}$ DMBA, orthotopically every week for 18 weeks. This study indicated that WT mice started to show tumour growth from the 7th week of DMBA treatment and 
about $90 \%$ of WT mice developed epithelial tumours (up to 6 tumours per mouse) at the end of the treatment period (Fig. 3a,b; Supplementary Table 3). In contrast, fewer than $20 \%$ of SKO mice developed tumours within the same time period. Moreover, of those $20 \%$, the number of tumours observed was $<3$ per animal and tumour growth was not observed until 14 weeks after DMBA treatment (Fig. 3a,b; Supplementary Table 3). Histological analysis of skin/tumour biopsies indicated that DMBA-treated
WT mice developed squamous cell carcinoma (SCC) (Fig. 3c; Supplementary Fig. 4a). CD68 and CD11c staining showed that DMBA-treated WT mice exhibited significant phagocytic infiltration and enhanced inflammation of the tumour/skin area (Fig. 3d; Supplementary Fig. 4b-d). Indeed, the majority of DMBA-treated SKO mice showed no evidence of tumour presence or enhanced inflammation (Fig. 3c,d; Supplementary Fig. $4 a-d)$. To evaluate cytokine production in the skin of the a
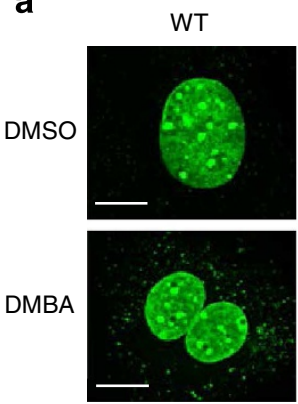

C
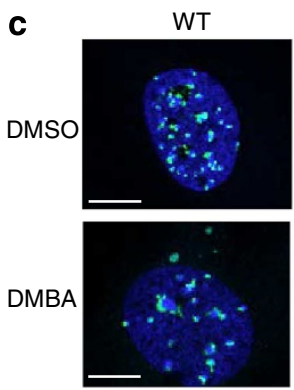

e
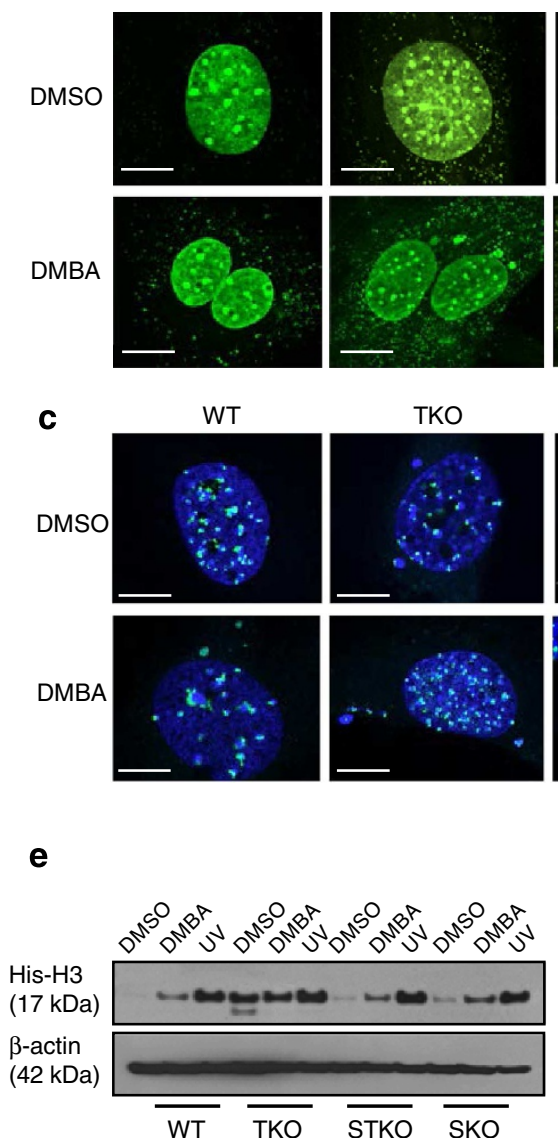

TKO

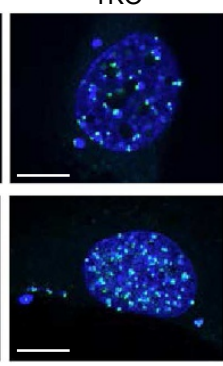

STKO

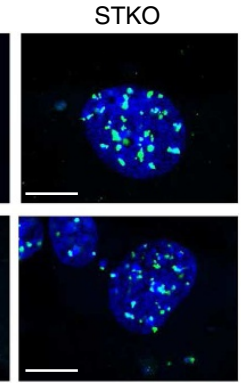

$\mathbf{f}$
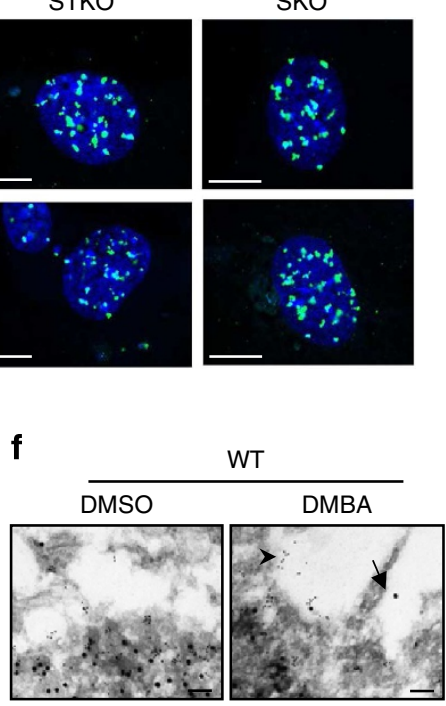

b

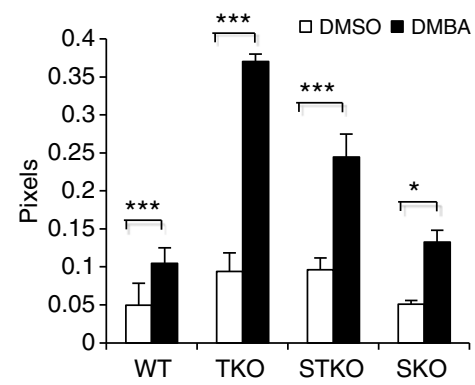

d

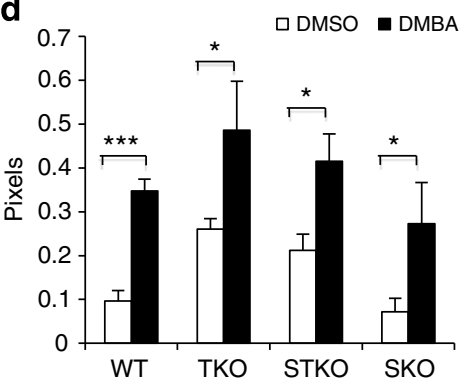

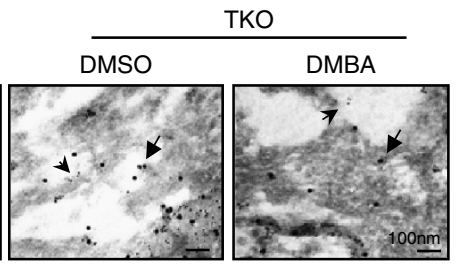

$6 \mathrm{~nm}$ Gold : anti-His-H3 $15 \mathrm{~nm}$ Gold : anti-dsDNA

Figure 2 | Cytoplasmic nucleosome production in DMBA-treated cells is independent of STING. (a) Fluorescence microscopy analysis of anti-dsDNA staining $(\mathbf{a}, \mathbf{b})$ and fluorescent in situ hybridization (FISH) with mouse centromere probe (c,d) in WT, TKO, STKO and SKO MEFs treated with DMBA at $20 \mu \mathrm{g} \mathrm{ml}^{-1}$ or DMSO as control for $48 \mathrm{~h}$. Representative images are shown at $\times 1,260$; bar size, $10 \mu \mathrm{m}$ (a,c), and ratio of cytoplasm to nucleus anti-dsDNA pixels from a and FISH pixels from c was quantitated using Leica LAS AF software (b,d). (e) Immunoblot analysis of histone H3 in cytoplasmic extraction from MEFs treated with DMBA same as in a or ultraviolet at $120 \mathrm{~mJ} \mathrm{~cm}^{-2}$ followed by $24-\mathrm{h}$ incubation (also see Supplementary Fig. 10). (f) Electron microscopy analysis of histone $\mathrm{H} 3$ and dsDNA gold staining in WT and TKO MEFs treated with DMBA same as in a. Arrows indicate gold particles in cytoplasm. Images were shown at original magnification, $\times 100,000 ;$ bar size, $100 \mathrm{~nm}$. Data are representative of at least two independent experiments. Error bars indicate s.d. ${ }^{\star} P<0.05,{ }^{\star \star \star} P<0.001$, Student's $t$-test.

Figure 3 | DMBA-induced skin carcinogenesis is mediated through STING. (a) Percentage of skin-tumour-free mice and (b) number of skin tumours from DMBA-treated WT $(n=18)$ and SKO mice $(n=16)$. Acetone is used as control treatment. Results are shown in the Kaplan-Meier curve for the representative of two independent experiments. $P$ value of tumour incidence between group 1 and group 2 was calculated from the log-rank test. (c) Representative photographs of skin tumours and haematoxylin/eosin staining of the mice same as in a; all images were shown at original magnification, $\times 200$; bar size, $200 \mu \mathrm{m}$. (d) CD68 immunohistochemistry staining of skin/tumours from mice same as in a; all images were shown at original magnification, $\times 400$; bar size, $100 \mu \mathrm{m}$. (e) Gene array analysis of skin/tumour RNA isolated from the mice same as in a and (f) fold change values. Highest variable inflammation-related genes are shown. Rows represent individual genes; columns represent individual samples. Pseudo-colours indicate transcript levels below (green), equal to (black) or above (red) the mean. Scale represents the intensity of gene expression (log2 scale ranges between -5 and 5). (g) Quantitative PCR analysis of IL19, IL24 and IL1 $\beta$ from RNA same as in e. Data are the mean of three independent mice. Error bars indicate s.d. ${ }^{\star} P<0.05$, Student's $t$-test. NS, not significant. 
treated WT or SKO mice, we performed DNA microarray analysis on tumours or skin derived from the treated animals. This study indicated extremely high levels of cytokine production in SCC derived from the DMBA-treated WT mice, including IL1 $\beta$, IL24, IL19, IL6 and CCL5, proinflammatory cytokines that have been shown to have profound effect in tumour development
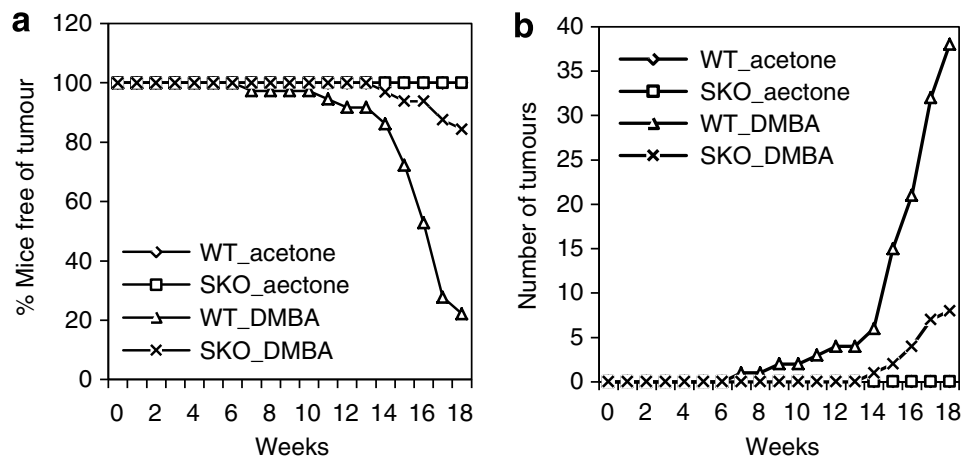

\begin{tabular}{|c|c|}
\hline Group 1 & Group 2 \\
\hline WT_acetone & WT_DMBA \\
\hline SKO_aectone & SKO_DMBA \\
\hline WT_DMBA & SKO_DMBA \\
\hline
\end{tabular}

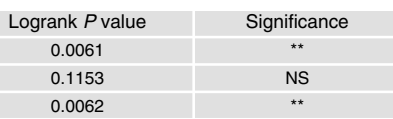

C
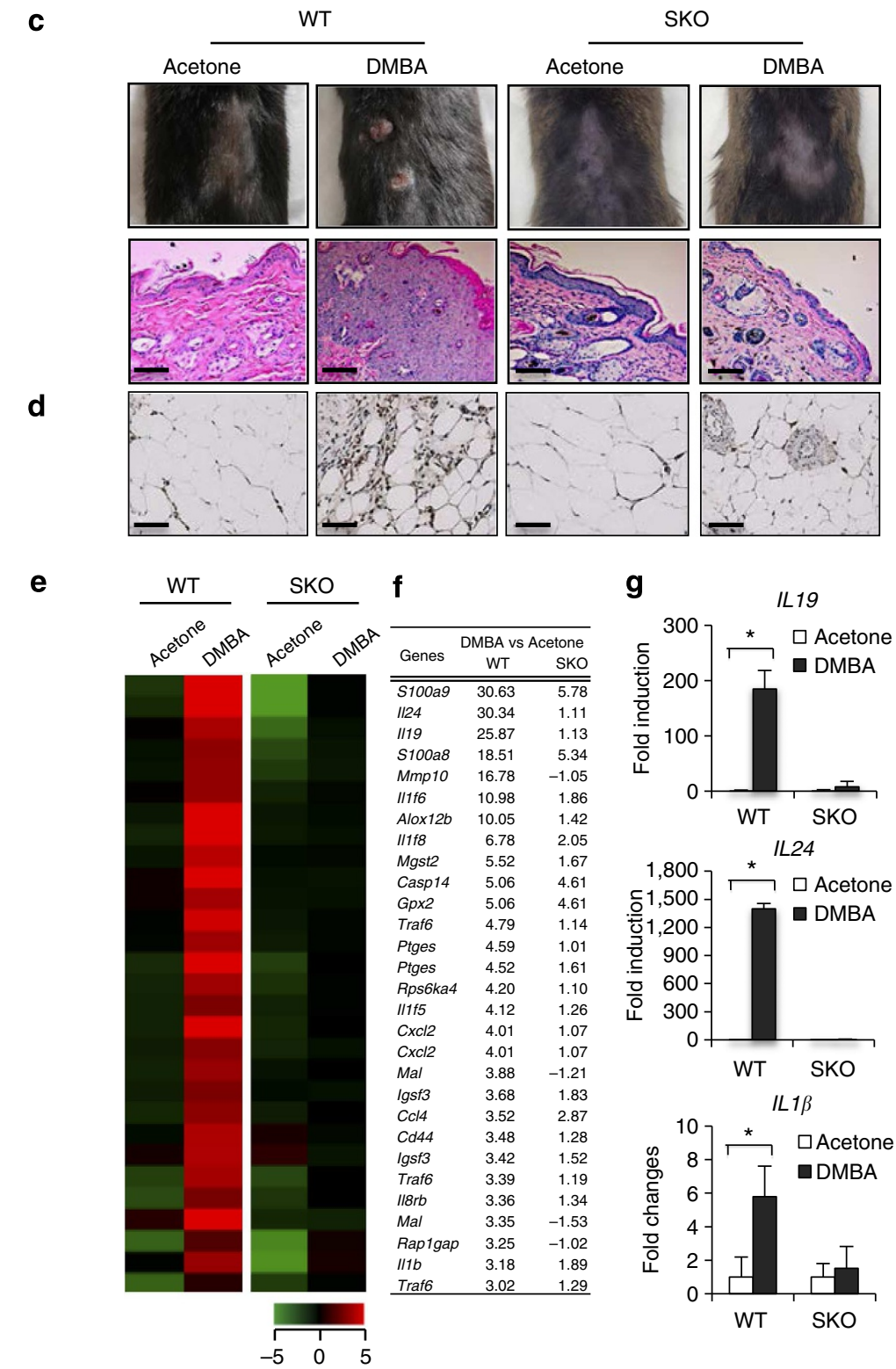
by numerous studies ${ }^{21-23}$ (Fig. 3e-g; Supplementary Fig. 4e,f). In contrast, the levels of these cytokines were significantly ablated in the skin of similarly treated SKO mice or mock-treated control mice (Fig. 3e-g; Supplementary Fig. 4e,f). Our data demonstrate that STING-dependent cytokine production is responsible for DMBA-dependent inflammatory carcinogenic disease. We similarly treated Trex1-deficient mice (TKO) with DMBA. All DMBA-treated TKO mice developed skin tumours as early as 9 weeks. Histological analysis of the skin derived from treated TKO mice showed markedly thickened epidermis with SCC extending into the dermis (Supplementary Fig. 5). Langerhans cells (LCs) have been reported to play a key role in manifesting DMBAfacilitated inflammatory tumours ${ }^{24,25}$. We observed that, upon DMBA treatment, WT skin/tumour showed a notable increase in LCs (using CD207 antibody) compared with SKO skin (Supplementary Fig. 6a,b). We also evaluated DMBA metabolic profile in WT versus SKO MEFs by high-pressure liquid chromatography (HPLC). Our data suggested that both MEFs are capable of metabolizing DMBA, which is consistent with the comparable level of DNA damage observed in DMBA-treated a

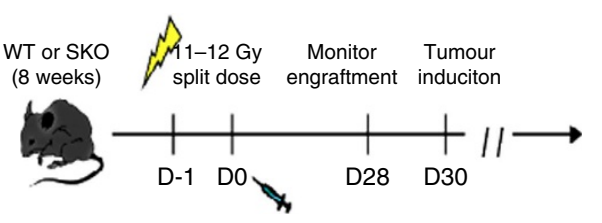

b

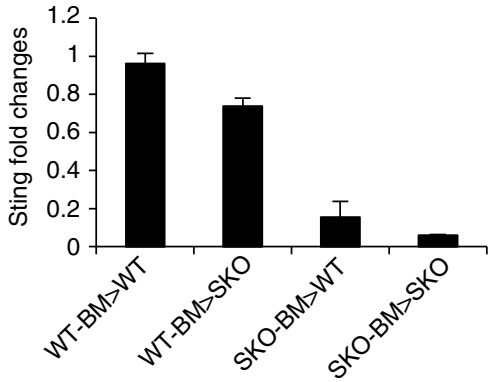

C

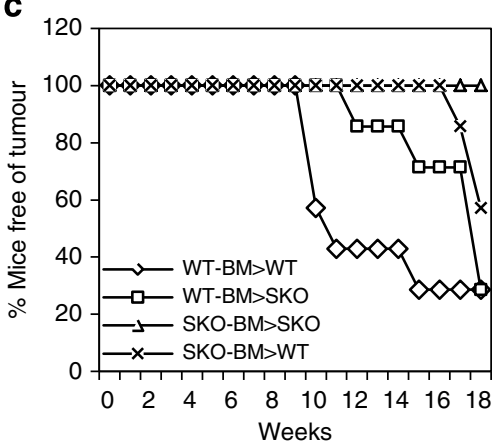

d

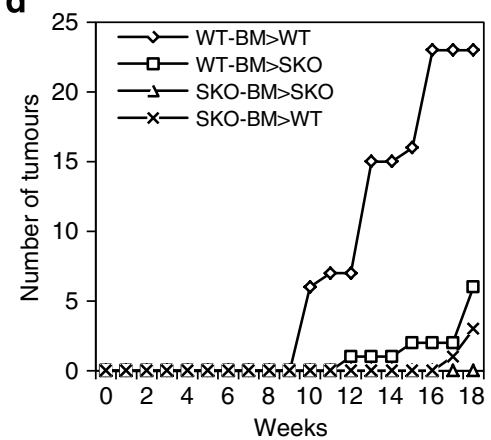

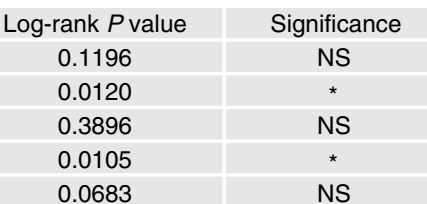

SKO

$\overline{\text { WT SKO }}$

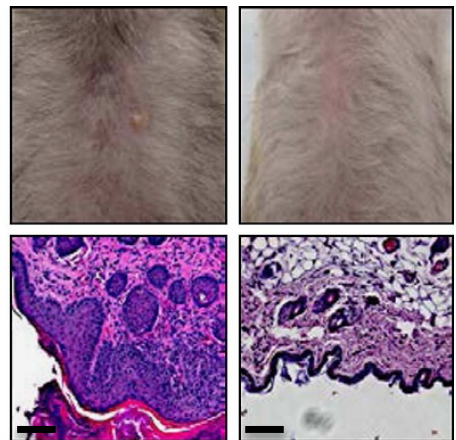

Figure 4 | STING in hematopoietic cells contributes to DMBA-induced carcinogenesis. (a) Schematic representation of bone marrow transplantation (BMT). (b) STING expression level in peripheral blood mononucleated cell 4 weeks post BMT. Transplant groups are labelled as: donor bone marrow $>$ recipient. (c) Percentage of skin-tumour-free mice and (d) number of skin tumours from DMBA-treated mice previously transplanted with bone marrow as indicated. $N=7$ per group. Results are shown in the Kaplan-Meier curve. $P$ value of tumour incidence between group 1 and group 2 was calculated from the log-rank test. (e) Representative photographs of skin tumours and haematoxylin/eosin staining of the mice same as in c. Images are shown at original magnification, $\times 200$; bar size, $200 \mu \mathrm{m}$. NS, not significant. 
WT and SKO MEFs (Supplementary Fig. 6c). Thus, DNAdamage-induced inflammatory carcinogenesis can be facilitated through the STING pathway.

STING in bone marrow contributes to DMBA skin tumorigenesis. To evaluate the role of hematopoietic cells (plausibly antigen-presenting cells) in DMBA-induced carcinogenesis, we irradiated 8-week-old WT or SKO mice and adoptively transferred them with bone marrow from WT or SKO mice. The transplanted mice were then treated with or without DMBA 28 days later for up to 20 weeks. This study showed that DMBAtreated WT mice previously transplanted with WT bone marrow exhibited a similar frequency of developing epithelial carcinoma compared with WT mice treated with DMBA alone (up to 9 tumours per mouse) (Fig. 4a-d). In contrast, WT mice transplanted with bone marrow obtained from SKO mice developed fewer tumours $(<2$ tumours per mouse), with $>60 \%$ of the animals remaining tumour free (Fig. $4 \mathrm{c}, \mathrm{d}$ ). It is plausible that the persistence of tumour development in some of the SKO bonemarrow-transferred WT mice is due to DMBA-induced cytokine production in radioresistant cells (for example, LCs, keratinocytes and so on) that are still positive for STING. More significantly, irradiated SKO mice treated with DMBA after transplantation with bone marrow from WT mice developed tumours to a similar frequency compared with irradiated, DMBA-treated WT mice receiving WT bone marrow (Fig. 4c,d). Histological examination indicated that both WT and SKO mice transplanted with WT bone marrow developed severe SCC, whereas the majority of WT and all SKO mice transplanted with SKO bone marrow showed no tumour or inflammation (Fig. 4e). Thus, we conclude that while carcinogen-treated fibroblasts or epithelial cells are able to recognize DNA damage and produce cytokines in a STINGdependent manner, it is likely that this event promotes the infiltration of phagocytes. These latter cells encounter the DNAdamaged cells and engulf them ${ }^{3,5}$. This event triggers further STING-dependent cytokine production, which predominantly drives inflammation-dependent tumorigenesis. Indeed, our studies indicated that macrophages more readily engulfed DMBA-treated cells compared with untreated cells, an effect that could stimulate cytokine production (Supplementary Fig. 7). A key objective for the future would include identifying which cells (that is, tissue resident keratinocytes or inflammatory residual dendritic cells, LCs or macrophages) are predominantly involved in STING-dependent cytokine production and skin tumorigenesis. This will require confirming which cells are involved in DMBA metabolism and discerning between tumour induction by such agents and the promotion of cancer by the inflammatory response.

\section{Discussion}

Our data thus indicate that chronic exposure to carcinogens that induce DNA adduct formation and breakage can cause nucleosome leakage and trigger cytosolic DNA signalling that is dependent on STING. This effect is greatly augmented in the absence of Trex1 (DNaseIII), indicating a possible role for this predominantly ER-associated exonuclease in eliminating selfDNA that has leaked into the cytoplasm, consequences that lead to the enhanced stimulation of STING (Supplementary Fig. 8). Loss of TNF $\alpha$ or MyD88 has also been shown to render mice resistant to DMBA treatment ${ }^{11,12}$. However, we have found that STING-dependent primary innate immune gene activation remains intact in MyD88 lacking MEFs (Supplementary Fig. 9). Thus, STING likely triggers the expression of select cytokines that in turn bind receptors requiring MyD88 signalling for the production of proinflammatory cytokines such as TNF $\alpha^{2}$. It is also plausible that drugs such as cisplatin and etoposide, given transiently, may exert some of their anti-tumour activity by triggering STING-dependent innate immune activity. These events could facilitate tumour clearance by stimulating antitumour-adaptive immune responses. It is further tempting to speculate that stimuli such as PAHs, that are predominant in numerous environmental toxins such as smoke and certain foods, could play a role, at least in part, for the inflammatory effects observed in patients with defects in genes such as Trex1, which can cause lethal AGS or less-severe types of SLE. Our data, which is the first report for a role of the STING pathway in cancer, suggest that controlling STING-dependent cytokine production could have beneficial effects on the outcome of certain malignant diseases. Thus, therapeutics that control STING may provide a new generation of anti-cancer treatments.

\section{Methods}

Materials. All cell culture media or reagents were from Invitrogen unless specified. All chemicals were from Sigma unless specified.

Mice. SKO mice were generated in our laboratory (Ishikawa et al. ${ }^{13}$ ). Trex1 hetero mice (Trex1 ${ }^{+/-}$) were kindly provided by Dr Lindahl and Barnes (London Research Institute). SKO mice were crossed with Trex $1^{+/-}$mice to generate STING and Trexl double-knockout mice (STKO). Mice care and study were conducted under approval from the Institutional Animal Care and Use Committee of the University of Miami. Mouse genotypes from tail biopsies were determined by using real-time PCR with specific probes designed for each gene by commercial vendor (Transnetyx).

Mouse treatment. Dorsal skin 1-2 $\mathrm{cm}^{2}$ of 8-week-old female mice was shaved. For treatment with DMBA, $10 \mu \mathrm{g}$ of DMBA in $100 \mu \mathrm{l}$ of acetone was applied to shaved skin once per week for $18-20$ weeks. Acetone $(100 \mu \mathrm{l})$ was applied to control groups. Tumour development was assessed weekly. A mouse was killed if tumours reached $1 \mathrm{~cm}$ in any diameter or became obviously invasive or ulcerated. Skin/tumour biopsies were stored or treated for specific applications.

Immunohistochemistry and histological analysis. Skin/tumour biopsies were fixed with $10 \%$ formalin for $72 \mathrm{~h}$ and tranferred to $70 \%$ ethanol for long-time storage. Haematoxylin/eosin staining and immunohistochemistry staining of CD68 (AbD Serotec) at 1:200 dilution were done by IHC world. CD207 (Thermo Scientific) at 1:200 dilution and CD11c at 1:400 dilution (Bioss USA) staining and histological examination were performed by Dr Phillip Ruiz.

Bone marrow transplantation. Bone marrow cells were primarily harvested from the long bones of donor mice by flushing with a 26-gauge needle. Single-cell suspensions were T-cell depleted using anti-Thy1.2 (HO-13.4 supernatant) antibody at $1: 5 \mathrm{vol} / \mathrm{vol}$ for $30 \mathrm{~min}$ on ice, followed by addition of $12 \%$ Low-Tox M rabbit complement (Cedarlane Labs), incubated at $37^{\circ} \mathrm{C}$ for $1 \mathrm{~h}$. Depletion efficiency was routinely $>95 \%$. Recipient mice were lethally irradiated with a ${ }^{137} \mathrm{Cs}$ source at 6.5 Gy each 1 day before (day -1$)$ and on the day of transplantation (day 0 ), and later injected intravenously with $1 \times 10^{6} \mathrm{~T}$-cell-depleted bone marrow cells Hematopoietic cell population was examined by flow cytometry 4 weeks later to ensure engraftment. Transplanted mice were then treated with DMBA as described.

Primary cells and drug treatments. Mouse embryonic fibroblasts (MEFs) were obtained from e13.5 embryos by a standard procedure. Normal human epidermal keratinocytes and culture media were purchased from Lonza. Unless specified, cells were treated with DMBA at $20 \mu \mathrm{g} \mathrm{ml}^{-1}$, cisplatin at $10 \mu \mathrm{M}$ or etoposide at $250 \mu \mathrm{M}$ for $48 \mathrm{~h}$. Dimethyl sulfoxide (DMSO) was used for control treatment. Ultraviolet treatment on MEFs was performed in a UVC500 UV crosslinker at $120 \mathrm{~mJ} \mathrm{~cm}^{-2}$ followed by 24 -h incubation.

Gene array analysis. Total RNA was isolated from cells or tissues using the RNeasy Mini kit (Qiagen). RNA quality was analysed using the Bionalyzer RNA 6000 Nano (Agilent Technologies). Gene array analysis was examined using the Illumina Sentrix BeadChip Array (Mouse WG6 version 2) at the Oncogenomics Core Facility, University of Miami. Gene expression profiles were processed and statistical analysis was performed at the Bioinformatics Core Facility, University of Miami. Briefly, raw intensity values from the Illumina array are uploaded on GeneSpring software from Agilent. Values are Quantile normalized and log2transformed to the median of all samples. Significantly differential expressed genes from a two-class comparison are computed using the Student's $t$-test and selected using a threshold of $P$-value $\leq 0.05$. Hierarchical clustering and visualization of 
selected differentially expressed genes is performed on GeneSpring using the Pearson correlation distance method, and linkage was computed using the Ward method. Fold change analysis was performed between two groups and differentially expressed genes were selected based on threshold of fold changes.

Cytoplasmic nucleosome detection. The cytoplasmic fraction was extracted from DMBA-treated MEFs using NE-PER nuclear and cytoplasmic extraction reagents (Thermo scientific). Equal amounts of proteins were analysed by immunoblot.

ELISA. IFN $\beta$ levels were determined from cell culture supernatant using the VeriKine Mouse IFN-Beta ELISA Kit (PBL IFN Source) following the manufacturer's instruction.

Quantitative real-time PCR. Total RNA was reverse-transcribed using the QuantiTect Reverse Transcription Kit (Qiagen). Real-time PCR was performed with the TaqMan gene Expression Assay (Applied Biosystems). mSTING-S (5'-CATTGGGTACTTGCGGTT- ${ }^{\prime}$ ), mSTING-AS (5'-CTGAGCATGTTGTTAT GTAGC-3'), mMyd88-S (5'-CACCAAGTCCTGGTTCTGG-3') mMyd88-AS (5'-TAGACAGGACGGCATCAGAG- $\left.3^{\prime}\right)$ was used for Sybr green analysis. Taqman gene expression assay: mouse CXCL10, Mm00445235; human CXCL10, Hs00171042; mouse IFNB, Mm00439552; Human IFNB, Hs00277188; mouse IL6, Mm00446190; mouse IL1B, Mm01336189; mouse cGAS, Mm01147497; mouse IL19, Mm01288324; mouse IL24, Mm00474102; mouse IRF1, Mm01288580, mouse TNFalpha, Mm00443258; mouse IRF7, Mm00516788; mouse CCL5, $\mathrm{m} 01302427$.

Immunofluorescence staining. Cells were fixed with $4 \%$ paraformaldehyde in Dulbecco's modified Eagle's medium for $15 \mathrm{~min}$ at $37^{\circ} \mathrm{C}$ and were permeabilized with $0.2 \%$ Triton X-100. Fixed and permeabilized cells were blocked with $10 \%$ bovine serum albumin in phosphate-buffered saline, incubated with primary antibodies in $2 \%$ bovine serum albumin in phosphate-buffered saline and then incubated with fluorophore-conjugated secondary antibodies (with $4^{\prime}, 6$-diamidino2-phenylindole (DAPI) counterstaining). After staining, cells were mounted in anti-fade mounting solution (Invitrogen) and examined under a Leica SP5 spectral confocal inverted microscope. Antibodies used including HA (Sigma) at 1:200 dilution, calreticulin (Abcam) at 1:200 dilution, IRF3 (Santa cruz) at 1:200 dilution, p65 (Cell signaling) at 1:200 dilution, dsDNA (Abcam) at 1:500 dilution and Histone $\mathrm{H} 3$ (Abcam) at 1:500 dilution.

PicoGreen staining. PicoGreen staining was performed using Quant-iT PicoGreen dsDNA reagent and kits from Invitrogen. For confocal microscopy, cells were incubated in the presence of PicoGreen at $37^{\circ} \mathrm{C}$ for $1 \mathrm{~h}$, washed and fixed for confocal microscopy with DAPI counterstaining.

Electron microscopy. After treatment, MEF cells were fixed in $4 \%$ paraformaldehyde. Histone $\mathrm{H} 3$ (Abcam, at 1:50 dilution) and dsDNA (Abcam, at 1:50 dilution) gold staining and electron microscopy analysis were performed by the electron microscopy core facility at Miami Project to Cure Paralysis, University of Miami Miller School of Medicine.

Fluorescence in situ hybridization. Cells were seeded on coverslips and treated appropriately. Fluorescence in situ hybridization was performed using the Cytology FISH accessory kit from DAKO following the manufacturer's instruction. Fluorescein isothiocyanate-labelled all-mouse centromere probe was purchased from Kreatech Diagnostics.

Bone marrow macrophage isolation and in vitro phagocytosis. Bone marrow macrophages were isolated from hind-limb femurs of 8-week-old WT and SKO mice. Briefly, the marrow cells were flushed from the bones with alpha minimum essential medium supplemented with $10 \%$ heat-inactivated foetal calf serum with a 26-gauge needle and incubated at $37^{\circ} \mathrm{C}$ for $4 \mathrm{~h}$. Approximately $2 \times 10^{7}$ cells were seeded in a 10-cm dish with complete alpha minimum essential medium including $10 \mathrm{ng} \mathrm{ml}^{-1}$ of mouse recombinant colony-stimulating factor (M-CSF, 416-ML) (R\&D Systems). After 1 week, bone marrow-derived macrophages were obtained. In vitro phagocytosis was performed as described by Ahn et al. ${ }^{5}$. Specifically, thymocytes were isolated from 8-week-old mice, treated with $20 \mu \mathrm{g} \mathrm{ml}^{-1}$ of DMBA for $48 \mathrm{~h}$ and transfected with fluorescein isothiocyanate-labelled dsDNA and then added to macrophages for $1 \mathrm{~h}$. Following vigorous washing to remove unengulfed thymocytes, macrophages were fixed with $4 \%$ paraformaldehyde solution overnight at $4{ }^{\circ} \mathrm{C}$, counterstained with DAPI and subjected to fluorescence microscopy analysis. For gene expression, macrophages were incubated with thymocytes for $6 \mathrm{~h}$ and subjected to quantitative PCR analysis.

Immunoblot analysis. Equal amounts of proteins were resolved on SDS-polyacrylamide gels and then transferred to polyvinylidene fluoride membranes (Millipore). After blocking with 5\% Blocking Reagent, membranes were incubated with various primary antibodies (and appropriate secondary antibodies). The image was resolved using an enhanced chemiluminescence system ECL (Thermo Scientific) and detected by autoradiography (Kodak). Antibodies: rabbit polyclonal antibody against STING was developed in our laboratory as described previously (Ishikawa et al. ${ }^{6}$ ); other antibodies were obtained from following sources: HA (Sigma Aldrich, at 1:10,000 dilution), $\beta$-actin (Sigma Aldrich, at 1:10,000 dilution), p-IRF3 (Cell Signaling, at 1:1,000 dilution), p-p65 (Cell Signaling at 1:1,000 dilution), p65 (Cell Signaling at 1:1,000 dilution), IRF3 (Santa Cruz Biotechnology, at 1:1,000 dilution), Histone $\mathrm{H} 3$ (Abcam at 1:1,000 dilution) and MyD88 (Abcam, at 1:500 dilution).

HPLC analysis of DMBA metabolism. Medium from treated MEFs as well as controls were collected and extracted with three equal volume of ethyl acetate. The organic extracts were evaporated to dryness with a Savant SpeedVac Evaporator/ Concentrator. The $\mathrm{pH}$ of the remaining aqueous fraction was lowered to 2.5 with $6 \mathrm{M}$ hydrochloric acid, and the contents were extracted again with ethyl acetate to isolate the acidic metabolites. The residues were dissolved in 30\% methanol for analysis by reversed-phase HPLC. DMBA and its metabolites were separated by HPLC using the Agilent Infinity 1260 LC system with a ZORBAX SB-C18 3.5-Micron $4.6 \times 150 \mathrm{~mm}$ column (Agilent) at a flow rate of $1 \mathrm{ml} \mathrm{min}^{-1}$. The compounds were eluted with a linear gradient of $40-95 \%$ methanol-water over $50 \mathrm{~min}$.

Statistical analysis. All statistical analyses were performed by the Student's $t$-test unless specified. The data were considered to be significantly different when $P<0.05$.

\section{References}

1. Grivennikov, S. I., Greten, F. R. \& Karin, M. Immunity, inflammation, and cancer. Cell 140, 883-899 (2010).

2. Trinchieri, G. Cancer and inflammation: an old intuition with rapidly evolving new concepts. Annu. Rev. Immunol. 30, 677-706 (2012).

3. Nagata, S., Hanayama, R. \& Kawane, K. Autoimmunity and the clearance of dead cells. Cell 140, 619-630 (2010).

4. Kawasaki, T., Kawai, T. \& Akira, S. Recognition of nucleic acids by patternrecognition receptors and its relevance in autoimmunity. Immunol. Rev. 243, 61-73 (2011)

5. Ahn, J., Gutman, D., Saijo, S. \& Barber, G. N. STING manifests self DNAdependent inflammatory disease. Proc. Natl Acad. Sci. USA 109, 19386-19391 (2012).

6. Ishikawa, H. \& Barber, G. N. STING is an endoplasmic reticulum adaptor that facilitates innate immune signalling. Nature 455, 674-678 (2008).

7. Burdette, D. L. \& Vance, R. E. STING and the innate immune response to nucleic acids in the cytosol. Nat. Immunol. 14, 19-26 (2013).

8. Gall, A. et al. Autoimmunity initiates in nonhematopoietic cells and progresses via lymphocytes in an interferon-dependent autoimmune disease. Immunity 36, 120-131 (2012).

9. DiGiovanni, J. Multistage carcinogenesis in mouse skin. Pharmacol. Ther. 54, 63-128 (1992)

10. Boffetta, P., Jourenkova, N. \& Gustavsson, P. Cancer risk from occupational and environmental exposure to polycyclic aromatic hydrocarbons. Cancer Causes Control 8, 444-472 (1997).

11. Moore, R. J. et al. Mice deficient in tumor necrosis factor-alpha are resistant to skin carcinogenesis. Nat. Med. 5, 828-831 (1999).

12. Cataisson, C. et al. IL-1R-MyD88 signaling in keratinocyte transformation and carcinogenesis. J. Exp. Med. 209, 1689-1702 (2012).

13. Ishikawa, H., Ma, Z. \& Barber, G. N. STING regulates intracellular DNAmediated, type I interferon-dependent innate immunity. Nature 461, 788-792 (2009).

14. Konno, H., Konno, K. \& Barber, G. N. Cyclic dinucleotides trigger ULK1 (ATG1) phosphorylation of STING to prevent sustained innate immune signaling. Cell 155, 688-698 (2013).

15. Yang, Y. G., Lindahl, T. \& Barnes, D. E. Trex1 exonuclease degrades ssDNA to prevent chronic checkpoint activation and autoimmune disease. Cell 131, 873-886 (2007).

16. Gehrke, N. et al. Oxidative damage of DNA confers resistance to cytosolic nuclease TREX1 degradation and potentiates STING-dependent immune sensing. Immunity 39, 482-495 (2013).

17. Kavanagh, D. et al. New roles for the major human $3^{\prime}-5^{\prime}$ exonuclease TREX1 in human disease. Cell Cycle 7, 1718-1725 (2008).

18. Greene, M. H. Is cisplatin a human carcinogen? J. Natl Cancer Inst. 84, 306-312 (1992).

19. Sun, L., Wu, J., Du, F., Chen, X. \& Chen, Z. J. Cyclic GMP-AMP synthase is a cytosolic DNA sensor that activates the type I interferon pathway. Science 339, 786-791 (2013)

20. Gabler, C. et al. Extranuclear detection of histones and nucleosomes in activated human lymphoblasts as an early event in apoptosis. Ann. Rheum. Dis. 63, 1135-1144 (2004). 
21. Chen, Y. Y., Li, C. F., Yeh, C. H., Chang, M. S. \& Hsing, C. H. Interleukin-19 in breast cancer. Clin. Dev. Immunol. 2013, 294320 (2013).

22. Lewis, A. M., Varghese, S., Xu, H. \& Alexander, H. R. Interleukin-1 and cancer progression: the emerging role of interleukin-1 receptor antagonist as a novel therapeutic agent in cancer treatment. J. Transl. Med. 4, 48 (2006).

23. Kumari, S. et al. Tumor necrosis factor receptor signaling in keratinocytes triggers interleukin-24-dependent psoriasis-like skin inflammation in mice. Immunity 39, 899-911 (2013).

24. Merad, M. et al. Langerhans cells renew in the skin throughout life under steady-state conditions. Nat. Immunol. 3, 1135-1141 (2002).

25. Modi, B. G. et al. Langerhans cells facilitate epithelial DNA damage and squamous cell carcinoma. Science 335, 104-108 (2012).

\section{Acknowledgements}

We thank Dr Biju Issac of the Sylvester Comprehensive Cancer Center Bioinformatic Core Facility for Gene expression array analysis; Ms. Delia Gutman and Ms. Auristela Rivera for mice breeding; Dr Robert Levy and Mr. Robert Newman for helping with bone marrow transplant; Dr Margaret Bates of the Electron Microscopy Core Facility at Miami Project to Cure Paralysis for EM analysis and Ms. Dayami Hernandez for helping with IHC.

\section{Author contributions}

J.A. and T.X. carried out most of the experiments. H.K. participated in animal experiment designing and performance. K.K. carried out qPCR and immunoblot analysis. P.R. provided histology examination and interpretation. G.N.B. wrote the manuscript.

\section{Additional information}

Accession codes: Gene array data has been deposited to GEO (Accession number GSE57605)

Supplementary Information accompanies this paper at http://www.nature.com/ naturecommunications

Competing financial interests: The authors declare no competing financial interests.

Reprints and permission information is available online at http://npg.nature.com/ reprintsandpermissions/

How to cite this article: Ahn, J. et al. Inflammation-driven carcinogenesis is mediated through STING. Nat. Commun. 5:5166 doi: 10.1038/ncomms6166 (2014). 